\title{
STEMI and NSTEMI ACS in a 30-Year-Old Patient: An Extremely Rare Complication of a Left Atrial Myxoma
}

\author{
Francesco Bartolomucci, MD, PhD,${ }^{1}$ Antonio Tito, $\mathrm{MD},{ }^{2}$ Eliano P. Navarese MD, PHD, ${ }^{3}$ \\ Fortunato Iacovelli, MD,${ }^{4 *}$ Marco Mele, MD,${ }^{5}$ Claudio Larosa, MD,${ }^{1}$ Marco M. Ciccone, MD ${ }^{2}$ \\ Mauro Cassese, MD, ${ }^{6}$ Martino Pepe, MD , $\mathrm{PhD}^{2}$ \\ ${ }^{1}$ Division of Cardiology, L. Bonomo Hospital, Andria (BT), Italy; ${ }^{2}$ Section of Cardiovascular Diseases, Department of \\ Emergency and Organ Transplantation, University of Bari, School of Medicine, Policlinico, Bari, Italy; ${ }^{3}$ Department of \\ Internal Medicine, Division of Cardiology, Pulmonology and Vascular Medicine, Heinrich-Heine-University, Düsseldorf, \\ Germany Systematic Investigation and Research on Interventions and Outcomes (SIRIO) MEDICINE research network, \\ Düsseldorf, Germany; ${ }^{4}$ Interventional Cardiology Service, "Montevergine” Clinic, Mercogliano, Italy; Division of \\ Cardiology, Department of Advanced Biomedical Sciences, University of Napoli "Federico II", Napoli, Italy; ${ }^{5}$ Coronary \\ Care Unit-Cardiology Unit, Teresa Masselli Mascia Hospital, San Severo (FG), Italy; ${ }^{6}$ Department of Cardiovascular \\ Surgery, Casa Sollievo della Sofferenza, San Giovanni Rotondo (FG), Italy
}

\section{ABSTRACT}

Primary cardiac tumors are a rare entity whose incidence in the general population ranges from $0.0017 \%$ to $0.28 \%$. Myxomas represent nearly half of all primary benign cardiac tumors and they prevalently affect female patients. Embolic manifestation is rare with a reported incidence of $0.06 \%$.

We present the case of a 30 -year-old male patient with acute anterolateral infarction caused by total occlusion of the left anterior descending artery as a consequence of a left atrial myxoma embolization. Urgent surgical resection of the mass didn't avoid early recurrence of atrial myxoma, whose second presentation was again myocardial infarction.

This case alerts physicians to "unusual" myocardial infarction patients, when atherosclerotic pathogenesis appears unlikely. In these cases early echocardiographic evaluation should be mandatory and atrial myxoma should be considered among the possible causes. Complete surgical resection is the only effective therapeutic option to improve prognosis; the chance of tumor recurrence should dictate careful research for additional myxomas during surgery and stricter follow-up planning.

\section{INTRODUCTION}

Primary cardiac tumors are a rare entity whose incidence in autopsy reports ranges from $0.0017 \%$ to $0.28 \%$ [McAllister 1978]. Myxomas represent nearly half of all primary benign cardiac tumors and they prevalently affect female patients between the third and sixth decades [Reynen 1996].

Received Fune 3, 2016; received in revised form November 23, 2016; accepted February 4, 2017.

*Fortunato Iacovelli is currently attending the Cardiopath PhD program, University of Napoli "Federico II", Napoli, Italy.

Correspondence: Martino Pepe, Section of Cardiovascular Diseases, Department of Emergency and Organ Transplantation, University of Bari, School of Medicine, Policlinico, Piazza Giulio Cesare 11, Bari, Italy; (e-mail: drmartinopepe@libero.it).
A non-negligible quote (about 20\%) [Lone 2008] of atrial myxomas are accidentally discovered in asymptomatic patients; nevertheless this tumor usually has three categories of manifestations: obstructive, systemic, and embolic. Obstructive symptoms depend on size, mobility, and localization of the mass and can frequently mimic mitral or tricuspid valve disease; systemic manifestations (fever, anemia, arthralgia, flushing, dyspnea) are believed to be caused by the direct tumor production of interleukins, by immunologic response, by bleeding and degeneration within the tumor, or by microembolism. Embolic manifestation is considered to be rare with a reported incidence of $0.06 \%$ and can be caused by fragmentation of the tumor mass or by embolization of thrombotic debris after thrombotic stratifications on the mass surface [Lehrman 1985].

\section{CASE REPORT}

We present the case of a 30-year-old male patient who accessed the emergency department because of sudden onset of dyspnea and chest pain. The only reported cardiovascular risk factor was a mild smoking habit. Vital signs were stable (BP was 120/80 $\mathrm{mmHg}$ and heart rate $90 \mathrm{bpm}$ ) and physical examination revealed no cardiac or other systemic abnormalities.

EKG showed 5-mm ST segment elevation in leads I, aVL, V3-V6 suggestive of acute anterolateral wall ST-elevation myocardial infarction (STEMI) (Figure 1, A); troponin I level was $9.37 \mu \mathrm{g} / \mathrm{L}$. Transthoracic echocardiography (TTE) revealed depressed systolic function (EF 40\%) driven by ipo/ akinesia of the apex and an ovalar pedunculated mass in the left atrium (Figure 1, C); the mass appeared attached to the free wall of the left atrium, almost completely hyperechoic, $45 \times 55 \mathrm{~mm}$ in size, and mobile with evident prolapse into the left ventricle (LV) during diastole. All these features were considered suggestive of left atrial myxoma.

The patient underwent urgent coronary angiography, which revealed the total occlusion of the distal segment of the left anterior descending artery (LAD) (Figure 1, B). Multiple 


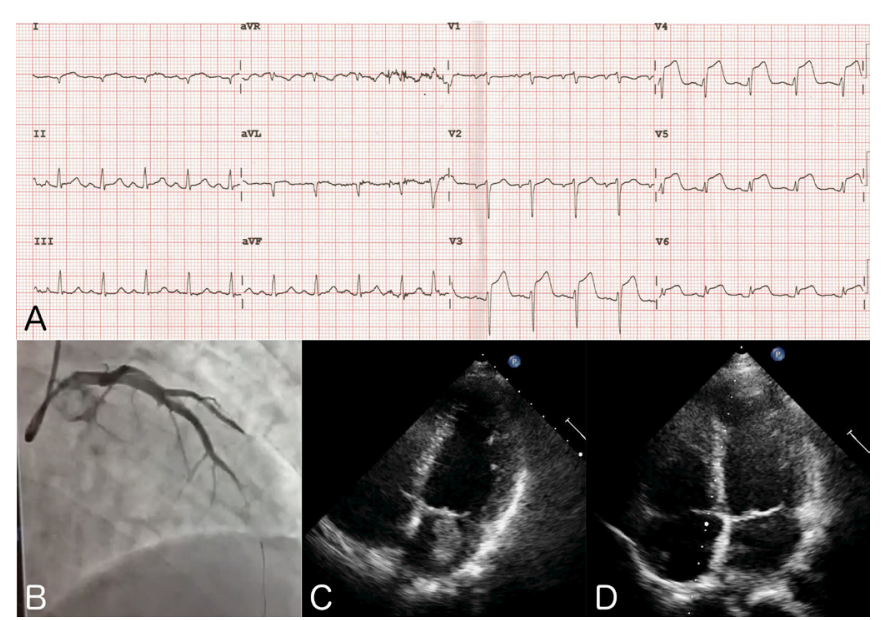

Figure 1. First myocardial infarction. A, EKG; B, coronary angiogram: total occlusion of the distal segment of LAD; C, transthoracic echocardiogram before surgery; $D$, transthoracic echocardiogram after surgery.

unsuccessful attempts of LAD mechanical reperfusion were done; the hypothesized non-thrombotic nature of the coronary occlusion discouraged thrombolytic therapy too. The patient was then referred to the cardiac surgeon, who performed the resection of the mass. A post-procedural TTE confirmed the excision of the mass (Figure 1, D) and the histological exam confirmed the diagnosis of atrial myxoma. Six days later the patient was dismissed asymptomatic.

Two months later the patient accessed again the emergency department complaining about the same symptoms: EKG showed negative T-waves in all the precordial leads (Figure 2, A) and troponin I level was $0.41 \mu \mathrm{g} / \mathrm{L}$. A second urgent coronary angiography was performed and showed a minus image at the middle segment of the LAD with distal TIMI flow 1 (Figure 2, B). A transesophageal echocardiography, performed afterwards, showed the recurrence of atrial myxoma, located between the left inferior pulmonary vein and left auricola, 11 $\times 10 \mathrm{~mm}$ in size (Figure 2, C and Figure 2, D); severe secondary mitral regurgitation and severe tricuspid regurgitation due to annular dilatation were also detected. The patient underwent a second surgery: resection of the tumor, tricuspid annular valvuloplasty, and coronary artery bypass grafting with left internal mammary artery to the LAD artery. A second histological exam confirmed the diagnosis of atrial myxoma.

\section{DISCUSSION}

The case we described is very peculiar in terms of epidemiology, clinical presentation, and prognosis.

As stated above, myxomas prevalently affect female patients between the third and sixth decade; despite these epidemiologic evidences, this case demonstrates myxomas can also occur in young male patients.

Embolic manifestation is considered to be rare and the most frequently targeted vessels are the cerebral arteries, followed by the peripheral and mesenteric ones. Embolism of coronary arteries, as in our case, is unusual probably because

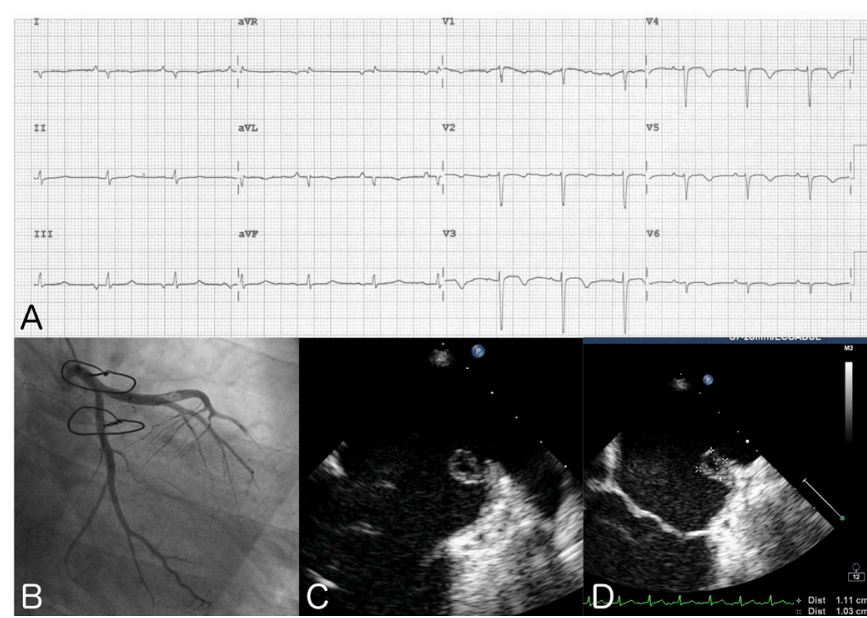

Figure 2. Second myocardial infarction. A, EKG; B, coronary angiogram: minus image at the middle segment of the LAD; $C$ and $D$, transesophageal echocardiogram.

of the perpendicular orientation of the coronary ostia and the protection offered during systole by aortic leaflets. Braun reported only 40 cases of myocardial infarction associated with atrial myxoma between 1970 and 2002 [Braun 2005]. Despite reported cases of spontaneous recanalization, tumor emboli may have malignant potential and can persist, invade and grow inside the coronary artery [Budzilovich 1979; Milicevic 2008]; moreover a predominance for the right coronary as the culprit vessel has also been shown. A possible explanation may be the orientation of the right coronary ostium relative to the aortic flow; nevertheless our case corroborates the idea that embolic events are a concrete possibility in the myxoma setting, and an acute myocardial infarction, also involving the LAD territory, cannot be ruled out.

The diagnosis of myxoma is usually made by 2D TTE; in the myocardial infarction scenario, cause time from first medical contact (FMC) to reperfusion is one of the more powerful prognosis determinants TTE is not mandatory and thus not always performed prior to coronary angiography: if that had happened in our case the myocardial infarction pathogenesis would have been missed with relevant therapeutic implications. For patients undergoing primary percutaneous coronary intervention (pPCI) the following dual antiplatelet therapy (DAPT) would drive an increased bleeding risk during the subsequent surgical resection of the mass, especially when surgery is not deferrable; moreover, despite pPCI currently being the gold standard treatment for myocardial infarction, thrombolysis is still an option for patients with an expected long "FMC to balloon" time and, if performed in this instance, it could easily cause further fragmentation of the tumor thrombotic stratification, potentially leading to massive systemic embolization [Abascal 1996].

The only therapy proven to improve prognosis of atrial myxoma in patients is early cardiac surgery [Demir 2005]; a six-monthly echocardiographic follow-up is recommended afterward [Reynen 1995]. In our case the scheduled followup was highly insufficient: an earlier reevaluation would have 
probably led to early myxoma recurrence recognition and have prevented complications.

Several hypotheses could have been proposed to explain tumor recurrence: inadequate resection, intraoperative implantation or embolization, multifocality, abnormal DNA ploidy, and malignant transformation; surgeons consequently need to perform a complete resection, avoiding embolization, but also carefully search for additional myxomas, through both transesophageal echocardiography and visual inspection. In our case, missing a second myxoma during the first resection cannot be ruled out; cases of multiple myxomas are also consistent with Carney's Complex: an autosomal dominant syndrome characterized by myxomas, primary pigmented nodular disease, testicular tumors, pituitary adenomas, schwannomas, and possibly other benign and malignant neoplasms. In our case, the absence of family members affected, and lack of other manifestations of the syndrome nevertheless make the latter hypothesis less likely [Shah 2015; Jones 1995].

\section{Conclusion}

It is the authors' opinion that when we face an "unusual" myocardial infarction patient, for instance very young and with few or no atherosclerotic risk factors, a bell should ring: we have to take into account non-atherosclerotic causes and these should include atrial myxoma. In these unusual cases, TTE should be considered mandatory and performed prior to any decision-making process about reperfusion therapy. We assessed that complete surgical resection is the only effective therapeutic option to improve prognosis, and the chance of tumor recurrence would suggest stricter follow-up planning in similar cases.

\section{REFERENCES}

Abascal VM, Kasznica J, Aldea G, Davidoff R. 1996. Left atrial myxoma and acute myocardial infarction. A dangerous duo in the thrombolytic agent era. Chest 109:1106-8.

Braun S, Schrotter H, Reynen K, Schwencke C, Strasser RH. 2005. Myocardial infarction as complication of left atrial myxoma. Int J Cardiol 101:115-21.

Budzilovich G, Aleksic S, Greco A. 1979. Malignant cardiac myxoma with cerebral metastases. Surg Neurol 11:461-9.

Demir M, Akpinar O, Acarturk E. 2005. Atrial myxoma: an unusual cause of myocardial infarction. Tex Heart Inst J 32:445-7.

Jones DR, Warden HE, Murray GF, et al. 1995. Biatrial approach to cardiac myxomas: A 30-Year clinical experience. Ann Thorac Surg 59:851-6.

Lehrman KL, Prozan GB, Ullyot D. 1985. Atrial myxoma presenting as acute myocardial infarction. Am Heart J 110:1293-5.

Lone RA, Ahanger AG, Singh S, et al. 2008. Atrial myxoma: trends in management. Int J Health Sci (Qassim) 2:141-51.

McAllister HA, Fenoglio JJ. 1978. Tumors of the cardiovascular systematlas of tumor pathology. 2nd series. Armed Forces Institute of Pathology, Washington DC, pp. 1-3 Fascicle 15.

Milicevic G, Gavranovic Z, Cupic H, Cerovec D, Stipic H, Jukic M. 2008. Unremitting embolus from cardiac myxoma at circumflex artery trifurcation. Int J Cardiol 126:424-6.

Reynen K. 1996. Frequency of primary tumors of the heart. Am J Cardiol 77:107.

Reynen K. 1995. Cardiac myxomas. N Engl J Med 333:1610-17.

Shah IK, Dearani JA, Daly RC. 2015. Cardiac myxomas: A 50-year experience with resection and analysis of risk factors for recurrence. Ann Thorac Surg 100:495-500. 